\title{
New Technique of Forensic Analysis for Digital Cameras in Mobile Devices
}

\author{
Jocelin Rosales Corripio, Ana Lucila Sandoval Orozco, Luis Javier García Villalba \\ Group of Analysis, Security and Systems (GASS) \\ Department of Software Engineering and Artificial Intelligence (DISIA) \\ Faculty of Information Technology and Computer Science, Office 431 \\ Universidad Complutense de Madrid (UCM) \\ Calle Profesor José García Santesmases, 9 \\ Ciudad Universitaria, 28040 Madrid, Spain \\ Email: jocelinr@ucm.es, \{asandoval, javiergv\}@fdi.ucm.es
}

\begin{abstract}
Nowadays, forensic analysis of digital images is especially important, given the high use of digital cameras in mobile devices. The identification of the device type or the make and model of image source are two important branches of forensic analysis of digital images. In this paper we have addressed both, with an approach based on different types of image features and the classification using support vector machines. The study mainly has focused on images created with mobile devices and as a result, the techniques and features have been adapted or created for this purpose. There have been a total of 36 experiments classified into 5 sets, in order to test different configurations of the techniques. In the configuration of the experiments were taken into account among other things the future use of the technique by the forensic analyst in real situations and creating experiments with high technical requirements.
\end{abstract}

Keywords-Forensics Analysis, digital image, image source acquisition identification, image noise features, image color features, image quality metrics, image wavelet features

\section{INTRODUCTION}

Currently, the demand for mobile devices (mobile phones, smartphones, tablets, etc.) increases year by year despite the global economic crisis. According to Gartner [1] in 2013 smartphone sales grew $42.3 \%$ over the previous year, outnumber for the first time the sales of feature phones. We must not overlook the emergence in today's society of such devices in our day to day life. Increasing storage capacity, usability, portability and affordability, have allowed mobile devices to be present in several activities, places and events of daily life. A consequence of its widespread use, is that digital images can be used as silent witnesses in judicial proceedings (child pornography, industrial espionage, ...), and in many cases crucial pieces of an evidence of a crime [2].

Forensic analysis of digital images can be mainly divided into two branches [3]: tamper detection and image source identification. This work focuses on the first branch. Also, since mobile device cameras have some characteristics that make them different from the rest, this work focuses on images from this type of devices. In this paper, we propose a method to image source acquisition in mobile devices. The objective of this approach is to identify make and model from a group the different images into disjoint sets in which all their images belong to the same device. This paper is structured into 5 chapters, being the first this introduction. The rest of the paper is structured as follows. Section 2 shows carries out a state of the art of techniques and algorithms for identifying the source type and source acquisition identification. Section 3 shows different sets of features (Noise, Color, Image Quality Metrics (IQM) and Wavelets) used by the algorithms and techniques of forensic analysis. In section 4, a set of experiments for the identification of device type and the source acquisition identification of the image are performed. In these experiments we use the set of the features previously presented and the algorithms of the techniques. Finally, section 5 shows the main conclusions of this work and some future work lines.

\section{RELATED WORK}

The main techniques of digital image forensics for identifying the source of image acquisition and the main work of the analysis. The success of these techniques depends on the assumption that all the images acquired by the same device have intrinsic features. The features which are used to identify the make and model of a digital camera are derived from the differences between the techniques of image processing technologies and the components which are used. The biggest problem with this approach is that different models of digital cameras use components of a small number of manufacturers, and the algorithms used are also very similar between models of the same brand. According to [4] for this purpose four groups of techniques can be established depending on their base: lens system aberrations, Color Filter Array (CFA) interpolation, image characteristics, and sensor imperfections. 
Techniques Based on Image Features use a set of features extracted from the content of the image to identify the source. These features are divided into three groups: color features, Image Quality Metrics (IQM) and wavelet domain statistics.

[5] proposes a method to identify the source using the following features: color features, image quality metrics and frequency domain. The study adopted the wavelet transforms as a method to calculate the wavelet domain statistics and use a Support Vector Machine (SVM) for classification. In experiments digital cameras and mobile devices were used. The results obtained in different experiments show results between $61.7 \%$ and $99.72 \%$ accuracy.

In [6] authors extend the source identification to different devices such as mobiles, phones, digital cameras, scanners and computers. In this proposal they base it on the differences in the image acquisition process to create two features groups: color interpolation coefficients and noise features. In the experiments they use five smartphone models, five digital camera models and four scanner models to identify the source type. Their experiments showed an overall result of $93.75 \%$ accuracy. Identifying the maker and model of five mobile phone models resulted in an accuracy of $97.7 \%$.

In [7] a method for source camera identification is proposed through the extraction and classification of wavelet statistical features. Finally 216 first-order wavelet features and 135 second order co-occurrence features is obtained. The most representative features are selected using an Sequential Forward Featured Selection (SFFS) algorithm and they are classified using a SVM. Identification success average of $98 \%$ the set of all cameras and an average success rate of $96.9 \%$ for the three cameras of the same model is achieved.

[13] performs experiments with common imaging features to identify the source: wavelet, color, IQM, statistical features of difference images and statistical features of prediction errors. In the experiments, different combinations of different types of features are used and a SVM for classification of different devices. Ten different cameras from four different makers with 300 images from each camera (150 for training and 150 for testing) and a resolution of $1024 \times 1024$ is used. Using all the features a score of $92 \%$ success rate is obtained. Moreover experiments were performed to check the robustness against three of the most common alterations in digital images: JPEG compression, cropping and scaling.

In [9] a technique for image source identification is proposed using ridgelets and contourlets subbands statistical models. After the feature extraction a SFFS algorithm is used for feature election and a SVM for classification. The method based on 216 wavelet features is considered useful only for the representation of a dimension, the approach based on ridgelets uses 48 features, and the approach based on contourlets includes a total of 768 features. In experiments with three cameras from different makers success rates are between $99.5 \%$ and $99.8 \%$.

In [10] a method using the marginal density Discrete Cosine Transform (DCT) coefficients in low-frequency coordinates and neighboring joint density features from the
DCT domain is proposed. Furthermore, hierarchical clustering and SVM is used to detect the source of acquisition of the images. In experiments with images from five smartphone models of four makers an accuracy of between $86.36 \%$ and $99.91 \%$ was obtained, achieving the best results with a linear SVM kernel.

\section{PROPOSED WORK}

Regarding classification, in [11] a study of different classification methods such as distance-based classifiers, Bayesian classifiers, neural networks, clustering algorithms and SVM classifiers is performed. As can be observed in the review, the use of SVM classifiers is widely used for these purposes. The kernel choice depends, among other factors, on the nature of the data to be classified. This paper will use an SVM classifier with Non-linear RBF kernel, as it is recommended for use when there is no a priori information about the data. The parameters for the SVM are the same as those used in [12]. Likewise, the option chosen is the most widely used one by the most recent precise works and they present good results. There are many implementations of SVM classifiers; particularly in this work we opted to use the LibSVM library [13].

The set of features to be used can be classified into four major groups, depending on the nature of their obtaining: noise features (16 features), color features (12 features), IQM (40 features) and wavelets (81 features). A detailed analysis on each of the aforementioned feature sets will be performed below.

\section{A. Noise Features}

One of the objectives is to get a set of features that allow us to differentiate between the different types of devices. To do this we firstly take into account that digital cameras use a twodimensional array sensor whereas most scanners use a linear array sensor. In the case of scanners, the linear arrangement of the sensor moves to generate the entire image, so it is expected to find the periodicity of the sensor noise within the rows of the scanned image. On the other hand, there is no reason to find sensor noise periodicity within the columns of the scanned image. In the case of digital cameras this type of noise periodicity does not exist. This difference can be used as a basis to discriminate between different types of devices. Noise features extraction is based on [14].

Let $I$ an image of $M \times N$ pixels, $M$ as the rows and $N$ as the columns. We denote $I_{\text {noise }}$ the noise of the original image and $I_{\text {noise }}$ is the image without noise.

$$
I_{\text {noise }}=I-I_{\text {denoise }}
$$

Then, each color component of the image without noise is subtracted to each color component of the original image, with which we obtain noise components of each pixel disaggregated for each color component.

The image original noise $I_{\text {noise }}$ can be modeled as the sum of two components, the constant noise $I_{\text {noiseconstant }}$ and random noise $I_{\text {noiserandom }}$. For scanners constant noise only depends of the column index, because the same sensor is 
moved vertically to generate the complete image. The average noise of all columns can be used as a pattern reference $I_{\text {noiseconstant }}(1, j)$ because the random noise components were cancelled. For detecting the similarity between different rows with the pattern reference, we use the correlation of these rows with the pattern.

$$
\operatorname{corr}(X, Y)=\frac{(X-\bar{X}) \cdot(Y-\bar{Y})}{\|X-\bar{X}\| \cdot\|Y-\bar{Y}\|}
$$

Then the same process is performed to detect the similarity of the columns with the pattern reference. After obtaining the correlation between rows and between columns we will go to obtain the feature set. It should be noted at the time of obtaining the features, that in the case of scanners the orientation of the image is critical, because features obtained will be completely different.

For each type of correlation first order statistical values are obtained, which are: mean, median, maximum and minimum. Also, the ratio features between rows and columns correlations are added. Finally the average noise per pixel feature was included. This feature does not depend on rows or columns correlations with the reference pattern, but is independent and it can distinguish between different types of devices, such as computer generated images. In total a set of 16 features are obtained: 7 rows features, 7 columns features, the ratio between rows and columns correlations and the average noise per pixel.

\section{B. Color Features}

The configuration of the CFA filters, the demosaicing algorithm and color processing techniques mean that signals in the color bands may contain treatments and specific patterns. In order to determine the differences in color features for different camera models, it is necessary to examine the first and second order statistics of the pictures taken with them.

- Pixels average value: This measure is performed for each RGB channels (3 features).

- Correlation pair between RGB bands: This measure expresses the fact that depending on the structure of the camera, the correlation between the different color bands can change ( 3 features which come from measuring the correlation between the RG, RB and GB bands).

- Neighbor distribution center of mass for each color band: This measure is calculated for each band separately (3 features). Firstly, the total number of pixels for each color value is calculated, obtaining a vector with 256 components. Then, with these calculated values the sum of neighboring values are obtained.

- Energy ratios between pairs RGB: This feature depends on the white dots correction process of the camera ( 3 features)

\section{Image Quality Metrics}

Different camera models produce images of different quality. There may be differences in image brightness, sharpness or quality color. These differences propose a set of quality metrics features that help us to distinguish the image source. There are different IQM categories: measures based on the pixels differences, measures based on correlation and measures based on spectral distance. For obtaining this set of metrics, a filtered image in which the noise of the original image is reduced to perform different calculations is needed in addition to the original image. For this, a Gaussian filter that allows us to perform image smoothing is used. After the core is obtained, it is normalized, so that the sum of all its components is 1 . This is necessary to obtain a smooth image but with the same colors as the original. The normalization is performed dividing each component by the sum of the values of all the components. For obtaining the metrics a filter with a $3 \times 3$ kernel with $\gamma=0.5$ is used. Following the specification of the 40 IQM features based on [8].

- Czekonowsky distance: The Czekonowsky distance is a useful metric for comparing vectors with no negative components as in the case of color images.

- Minkowsky metrics: Minkowsky metrics for $\gamma=1$ and $\gamma=$ 2.

- Normalized Cross Correlation: The closeness between two digital images can also be quantified in terms of a correlation function. The quality metric of the normalized cross-correlation measurement for each image band $k$.

- Structural Content: The structural content of an image quality metric is defined for each band $k$.

- Spectral Measures: The Discrete Fourier Transform (DFT) of the original image and the smoothed image, denoted as $\tau_{k}(u, v)$ and $\hat{\tau}_{k}(u, v)$ for a band $k$.

- Measures based on the human visual system: Images can be processed by filters which simulate the Human Visual System (HVS). One of the models used for this is a bandpass filter with a transference function in polar coordinates.

\section{Wavelet Features}

Due to the deterministic property of the sensor pattern noise which is present in an image, this pattern can be used as a footprint to identify the device that generated the image under investigation. It can be said that the sensor pattern noise is to a digital camera as a fingerprint is to a human being. To identify the acquisition source we require an algorithm that allows us to extract the sensor noise and another that allows us to obtain the features of the fingerprints obtained in order to classify and identify them.

Taking the main ideas from [15] as a reference, algorithm 1 is proposed to extract sensor noise.

\begin{tabular}{lc}
\hline Algorithm 1: Extracting PRNU \\
\hline 1. & Apply a wavelet decomposition in 4 levels to I; \\
2. & Foreach wavelet decomposition level do \\
3. & Foreach component c $\epsilon$, $V, D\}$ do \\
4. & Compute the local variance; \\
5. & If (adaptive variance) \\
6. & Compute 4 variances with windows of size: 3, \\
7. & 5,7 and 9 respectively; \\
8. & Select the minimum variance; \\
9. & else \\
10. & Compute the variance with a window of size $3 ;$ \\
& Compute noiseless wavelet components applying
\end{tabular}


11. Obtain $I_{\text {clean }}$ by applying the inverse wavelet transform with clean components calculated;

12. Obtain the sensor noise with $I_{\text {noise }}=I-I_{\text {clean }}$;

13. Apply zero-meaning to $I_{\text {noise }}$;

14. Increase the green channel weight with

$I_{\text {noise }}=0.3 \cdot I_{\text {noise }_{R}}+0.6 \cdot I_{\text {noise }_{G}}+0.3 \cdot I_{\text {noise }_{B}}$;

Finally, a total of 81 features (3 channels x 3 wavelet components $\times 9$ central moments) are calculated using algorithm 2 .

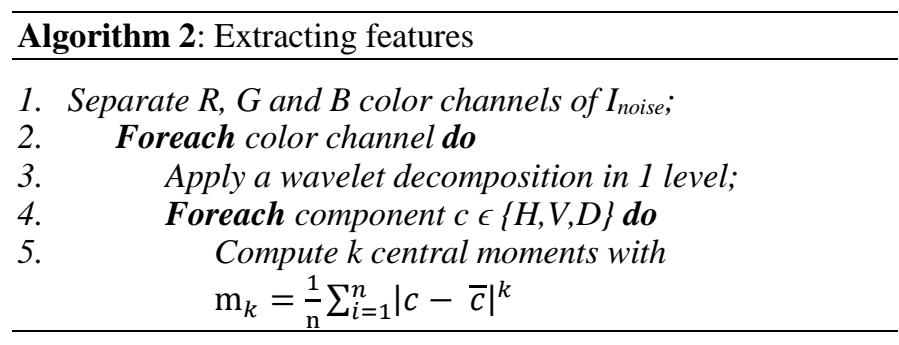

\section{EXPERIMENTS AND RESULTS}

We performed the classification of images on closed set of elements, i.e., the classes of the elements used in training are the same classes as those used in the test. The images used in the training stage are not used in the testing stage.

In order to evaluate the source device type identification we will use an image set composed of: images from mobile phones, images obtained from a scanner, and a computergenerated images. 200 images are used from each set, 100 for the SVM training and 100 for testing. All images have a resolution higher than $1024 \times 768$. There is no restriction on the content of the image or the camera configuration parameters at the time of the acquisition.

Images from 7 smartphones: IPhone 4s (I1), Blackberry 8520 (BB), Huawei U8815 (HU), LG P760 (LG2), Nokia 800 (N1), Samsung GT-I9001 (S1) and Sony C2105 (SE1). For images from scanners and computer-generated images, our own sources and the Flickr website were used. As a second filter for scanned images, those which had the tag "scanned images" and made reference to a retail scanner model were used. For the experiments we have taken into account the following configuration parameters: size of crop applied to the image, crop position (centered or upper-left corner) and application of different feature sets (Noise Features, Color Features, IQM Features and Wavelet Features).

Table I shows the results of success rates to evaluate the source device type identification between Camera (A), Computer (B) and Scanner (C), and the configuration parameters used in the 10 experiments.

From the analysis of the results, general and specific conclusions about the various configurations used in each experiment can be obtained. Encompassing all the experiments, it is observed that success rates are not excessively high ( $60.42 \%$ on average and $71.30 \%$ in the best case); it can be concluded that this technique is not particularly suitable for this purpose. It is important to emphasize, as noted above, that the number of different makes and models used for this experiment is high, which predictably causes success rates to drop. That being said, it should be noted that this study does provide interesting results on the configuration parameters used, since between the best and the worst result there is a difference in the average success rate of $23.48 \%$.

TABLE I. TPR WITH EQUAL NUMBER OF DEVICES THAN CLUSTERS

\begin{tabular}{|c|c|c|c|c|c|c|}
\hline \multirow{2}{*}{ Features } & \multirow{2}{*}{ Crop Size } & \multirow{2}{*}{ Crop Align } & \multicolumn{3}{|c|}{ Device (\%) } & \multirow{2}{*}{$\begin{array}{c}\text { Average } \\
(\%)\end{array}$} \\
\hline & & & A & $\mathrm{B}$ & $\mathrm{C}$ & \\
\hline \multirow{7}{*}{ Noise } & Full Size & - & 70 & 54 & 57 & 59.95 \\
\hline & $1024 \times 768$ & \multirow{3}{*}{ Center } & 66 & 80 & 46 & 62.39 \\
\hline & $800 \times 600$ & & 76 & 60 & 49 & 60.68 \\
\hline & $640 \times 480$ & & 62 & 61 & 48 & 56.62 \\
\hline & $1024 \times 768$ & \multirow{3}{*}{ Upper-left corner } & 76 & 59 & 40 & 56.40 \\
\hline & $800 \times 600$ & & 65 & 38 & 44 & 47.72 \\
\hline & $640 \times 480$ & & 74 & 54 & 37 & 52.88 \\
\hline \multirow{3}{*}{$\begin{array}{c}\text { All } \\
\text { Features }\end{array}$} & $1024 \times 768$ & \multirow{3}{*}{ Center } & 66 & 73 & 72 & 70.26 \\
\hline & $800 \times 600$ & & 69 & 74 & 71 & 71.30 \\
\hline & $640 \times 480$ & & 77 & 73 & 63 & 70.75 \\
\hline \multicolumn{3}{|c|}{ Average } & 69.9 & 61.3 & 51.4 & 60.42 \\
\hline
\end{tabular}

Given the importance of mobile images today, below we will show the experiment performed to identify the acquisition source of images from mobile devices, i.e., the classification of an image set according to the make and model of the camera that generated them.

The results improve significantly when all the features to identify the source type are used. Given the high number of classes, the results can be qualified as acceptable, since the average success rate for all experiments carried out using these features is $70.77 \%$. The experiments have been grouped into 3 groups with the aim of obtaining conclusions on: the use of different feature sets, crop size, the number of devices used for the classification, and the use of devices from the same manufacturer.

Table II shows the experiments in which 7 models of mobile devices from different manufacturers are used. Different types of combinations of features sets were tested. Most experiments were performed with a crop size of $1024 \times 768$, since as this is considered a large enough size to obtain good results, as shown in the previous experiments.

TABLE II. TPR WITH EQUAL NUMBER OF DEVICES THAN CLUSTERS

\begin{tabular}{|c|c|c|c|c|c|c|c|c|c|c|}
\hline Features & Crop Size & Crop Align & I1 & HU & LG2 & N1 & BB & S1 & SE1 & Average \\
\hline All Features (Daubechies 8-tap) & $1024 \times 768$ & Center & 93 & 96 & 80 & 94 & 91 & 70 & 85 & 86.54 \\
\hline
\end{tabular}


ICIT 2015 The $7^{\text {th }}$ International Conference on Information Technology

doi:10.15849/icit.2015.0105 C ICIT 2015 (http://icit.zuj.edu.jo/ICIT15)

\begin{tabular}{|c|c|c|c|c|c|c|c|c|c|c|}
\hline Noise & $1024 \times 768$ & Center & 41 & 42 & 35 & 18 & 40 & 40 & 62 & 37.67 \\
\hline Color & $1024 \times 768$ & Center & 24 & 37 & 20 & 40 & 31 & 19 & 44 & 29.27 \\
\hline IQM & $1024 \times 768$ & Center & 13 & 88 & 46 & 89 & 7 & 34 & 2 & 21.65 \\
\hline Wavelet Daubechies 8-tap & $1024 \times 768$ & Center & 95 & 96 & 96 & 94 & 92 & 76 & 93 & 91.46 \\
\hline Wavelet Haar & $1024 \times 768$ & Center & 95 & 87 & 97 & 70 & 86 & 56 & 91 & 81.84 \\
\hline Color + IQM + Wavelet Daubechies 8-tap & $1024 \times 768$ & Center & 93 & 94 & 90 & 90 & 90 & 53 & 85 & 83.67 \\
\hline All Features (Daubechies 8-tap) & $800 \times 600$ & Center & 91 & 96 & 84 & 92 & 95 & 56 & 85 & 84.41 \\
\hline All Features (Daubechies 8-tap) & $640 \times 480$ & Center & 90 & 95 & 84 & 89 & 88 & 51 & 88 & 82.15 \\
\hline
\end{tabular}

The experiment reveals that noise, color and IQM feature sets are individually completely invalid, since the best result obtains an $37.67 \%$ average success rate, which is unacceptable. With the remaining set of features (wavelets), two experiments were conducted using different types of wavelet: Daubechies 8tap and Haar. The results show that Daubechies 8-tap obtains better results than Haar and the best results of all experiments (91.46\%).

With respect to the different feature combinations, it is observed that when we use all the features good results are obtained (86.54\% in the best case), since, although they are slightly worse than the best result, the difference is not very significant $(4.92 \%)$. Also, the success rate when all the features are used subtly drops the smaller the crop size gets.

The combination of all the features except noise features, which are mainly focused on identifying the source type, yields an average success rate of $83.67 \%$. These results, even if not bad, are far from those obtained with the wavelets and worse than when the combination of all features is used.

\section{CONCLUSIONS}

In this work we have presented various techniques for identifying mobile device images with respect to scanned and computer-generated images. Besides, other techniques that allow us to distinguish the acquisition source of smartphone images are presented. The techniques are based on the use of four feature sets (Noise, Color, IQM and Wavelets), on which adjustments have been made in order to improve the results for this specific type of devices. There have been experiments with the combination of the different feature sets, different crop sizes and positions, and wavelet functions. With regard to source type identification, the first general conclusion is that Noise features are discarded as invalid when the number of types of devices is greater than 2. In the experiments that used whole images and different crop sizes and positions, unacceptable results were obtained for identifying three types of devices (scanner, smartphone and computer). As discussed in the experiments, for these three types of devices there are dozens of different manufacturers and models, hampering classification. As a counterpart, forensic analysts may consider the application of the technique with Noise features for identifying the source type of images from mobile devices with respect to images from scanners and computers. The results are quite good at identifying the type when discerning between scanners and smartphones. The use of all the features significantly improves results, but as a general conclusion they are not good enough to be used in a serious situation. When identifying the acquisition source of mobile device images, the results are much more encouraging. In all sets of experiments performed, there is at least one configuration that yields good results, always putting them into the context of the level of demand on this technique (a large number of devices or many devices from the same manufacturer).

\section{ACKNOWLEDGMENT}

The research leading to these results has been partially funded by the European Union's H2020 Program under the project SELFNET (671672). Part of the computations of this work was performed in EOLO, the HPC of Climate Change of the International Campus of Excellence of Moncloa, funded by MECD and MICINN. This work was supported by the "Programa de Financiación de Grupos de Investigación UCM validados de la Universidad Complutense de Madrid - Banco Santander".

\section{REFERENCES}

[1] Gartner Says Smartphone Sales Grew 46.5 Percent in Second Quarter of 2013 and Exceeded Feature Phone Sales for First Time (2013). URL http://www.gartner.com/newsroom/id/2665715

[2] M. Al-Zarouni, "Mobile Handset Forensic Evidence: a Challenge for Law Enforcement", in Proceedings of the 4th Australian Digital Forensics Conference, School of Computer and Information Science, Edith Cowan University, 2006.

[3] T. Gloe, M. Kirchner, A. Winkler, R. Bohme, "Can We Trust Digital Image Forensics?", in Proceedings of the 15th International Conference on Multimedia, ACM Press, 2007, pp. 78-86.

[4] T. Van Lanh, K. S. Chong, S. Emmanuel, M. S. Kankanhalli, "A Survey on Digital Camera Image Forensic Methods", IEEE International Conference on Multimedia and Expo, IEEE, 2007, pp. 16-19.

[5] M. J. Tsai, C. L. Lai, J. Liu, "Camera/Mobile Phone Source Identification for Digital Forensics",in Proceedings of the International Conference on Acoustics Speech and Signal Processing, IEEE, 2007, pp. II-221-224.

[6] C. Mckay, A. Swaminathan, H. Gou, M. Wu, "Image Acquisition Forensics: Forensic Analysis to Identify Imaging Source", in Proceedings of the IEEE International Conference on Acoustics, Speech and Signal Processing (ICASSP), IEEE, 2008, pp. 1657-1660.

[7] B. Wang, Y. Guo, X. Kong, F. Meng, "Source Camera Identification Forensics Based on Wavelet Features", in Proceedings of the International Conference on Intelligent Information Hiding and Multimedia Signal Processing, IEEE Computer Society, 2009, vol. 0, pp. 702-705.

[8] Y. Hu, C. T. Li, C. Zhou, "Selecting Forensic Features for Robust Source Camera Identification", Computer Symposium (ICS), 2010 International, 2010, pp. 506-511.

[9] L. Ozparlak, I. Avcibas, "Differentiating Between Images UsingWavelet-Based Transforms: A Comparative Study", IEEE Transactions on Information Forensics and Security, IEEE, 2011, vol. 6, no. 4 , pp. $1418-1431$. 
[10] Q. Liu, X. Li, L. Chen, H. Cho, A. P. Cooper, Z. Chen, M. Qiao, A. H. Sung, "Identification of Smartphone-Image Source and Manipulation", Advanced Research in Applied Artificial Intelligence, Lecture Notes in Computer Science, Springer Berlin Heidelberg, Dalian, China, 2012, vol. 7345, pp. 262-271.

[11] D. Michie, D. J. Spiegelhalter, C. C. Taylor, "Machine Learning, Neural and Statistical Classification", Ellis Horwood, 1994.

[12] J. Rosales Corripio, D. M. Arenas González, A. L. Sandoval Orozco, L. J. García Villalba, J. C. Hernandez-Castro, S. J. Gibson, "Source Smartphone Identification Using Sensor Pattern Noise and Wavelet Transform", In Proceedings of the 5th International Conference on
Imaging for Crime Detection and Prevention (ICDP 2013), pp. 1-6 2013

[13] C. C. Chang, C. J. Lin, "LIBSVM: A Library for Support Vector Machines". Version 3.17, Abril 26, 2013. URL http://www.csie.ntu.edu.tw/ cjlin/libsvm/

[14] N. Khanna, A. K. Mikkilineni, E. J. Delp, "Scanner Identification Using Feature-based Processing and Analysis", IEEE Transactions on Information Forensics and Security, IEEE, 2009, vol. 4, no. 1, pp. 123139.

[15] J. Lukas, J. Fridrich, M. Goljan, "Digital Camera Identification from Sensor Pattern Noise", IEEE Transactions on Information Forensics and Security, IEEE, 2006, vol. 1, no. 2, pp. 205-214,. 